\title{
The Research on Positions Distribution in Airport Operation Command Simulation System
}

\author{
Mou Qi-feng ${ }^{1, a}$,Sun Xi-ren ${ }^{1, b}$
}

${ }^{1}$ Air Traffic Management Department, Civil Aviation Flight University of China, Guanghan City, Sichuan Prov., China, 618307

E-mail: amouqifeng@sina.com, bsunnysunxiren@yahoo.com.cn

Keywords: Airport, Operation Command, Information Balance, Fuzzy Clustering, Positions Distribution

Abstract. This paper proposes a new position distribution method-Information Flow Configuration Method Based on Fuzzy Clustering Analysis, aiming at improving the precision and reliability of the airport operation command simulation system. It analyzed samples through the fuzzy clustering method, with the operation command information used as the clustering samples, and 7 kinds of information attribute used as clustering index. Then by using Matlab, it realized the cluster of the command information, and the cluster pedigree chart was drawn as well. At last, the correlation coefficient confirms that this approach is feasible in distributing the positions of the simulation system.

\section{Forward}

The airport operation command center is the key to run an airport, which is in charge of carrying organization, command, cooperation, control and emergency assistance ${ }^{[1]}$. The airport operation command simulation system is a particular emulation training system on account of the airport operation (see Figure 1), by which can give the airport operation controllers more practical training to improve their occupational skills. This system can help us to realize the goal of "low cost, high efficiency, wide range", and can provide the technical support cultivate and train professionals ${ }^{[2-3]}$.

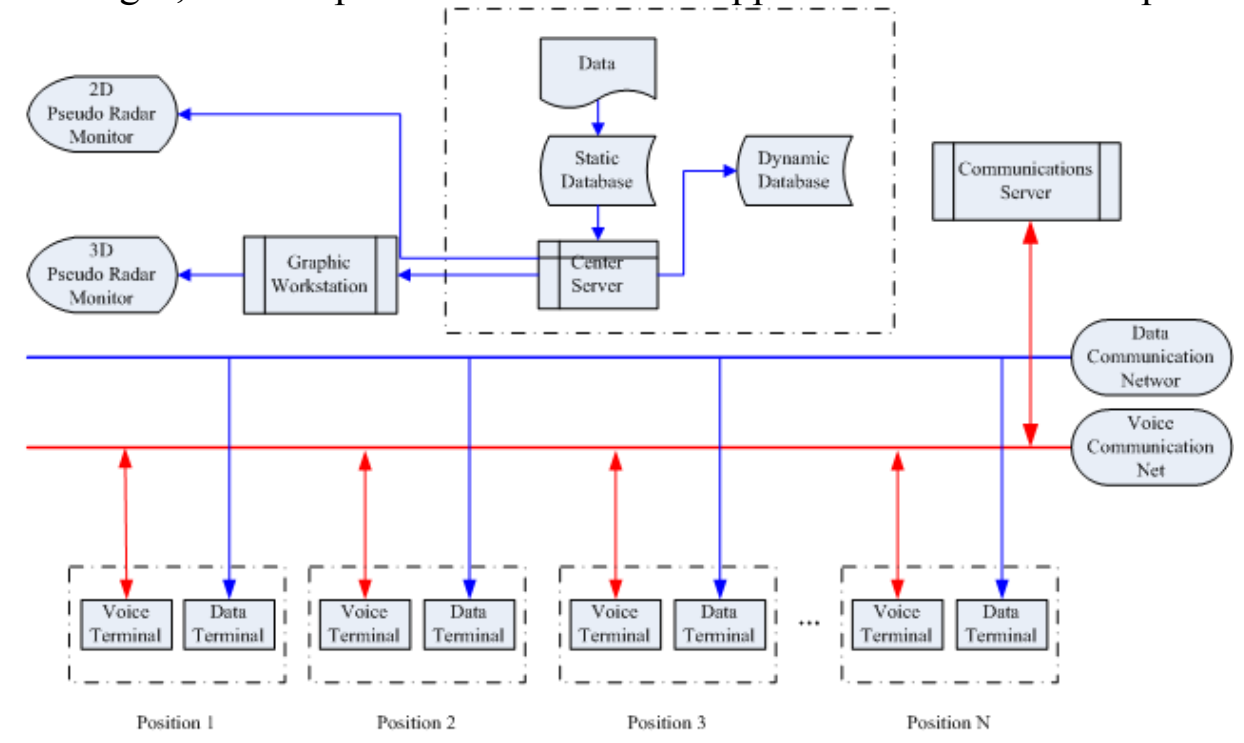

Fig.1 Topological graph of airport operation command simulation system

The rationalization of the responsibilities or seats distribution of the airport operation command simulation system directly affects the precision and reliability of this system used for simulation training. However, nowadays positions arrangement are varied from airport to airport, even the ICAO has no united criteria. Therefore, it is critical to propose a new command seats distribution method in order to promote the construction of airport operations command simulation system.

In this paper, the positions of airport operation command simulation system is divided by using fuzzy cluster analysis based on the equilibrium configuration of the information. According to the clustering results, a scheme of command positions distribution is proposed for decision-making. 


\section{Fuzzy clustering of command seats based on the balanced information}

Among the methods of classification, cluster analysis is a widely applied one. It sorts out different kinds of a group by characters, and puts those of the similar treats into one category, which guaranteed a highly identical quality of every one in a category and vice versa. In practice, boundaries among categories are vague; therefore, it is more subjective in applying the fuzzy mathematic principle to deal with the clustering ${ }^{[4]}$. And in this paper, the fuzzy clustering based on the balanced information is applied to solve the positions distribution of the airport operation command simulation system.

Hierarchical classification of operation command information. Considering the characteristics of the airport operation command system and the coordination of the multiple terminals ${ }^{[5]}$, we analyze and discuss the command information flow by using the system approach, according to the principle of layered concentrating, independent operation relatively. And we divide the command information into 21 categories as the clustering samples (see Figure 2), and choose 7 factors (flight production management, passenger service management, safety monitoring management, facilities instrument management, production control, emergency rescue, aeronautical information issued) as the clustering index.

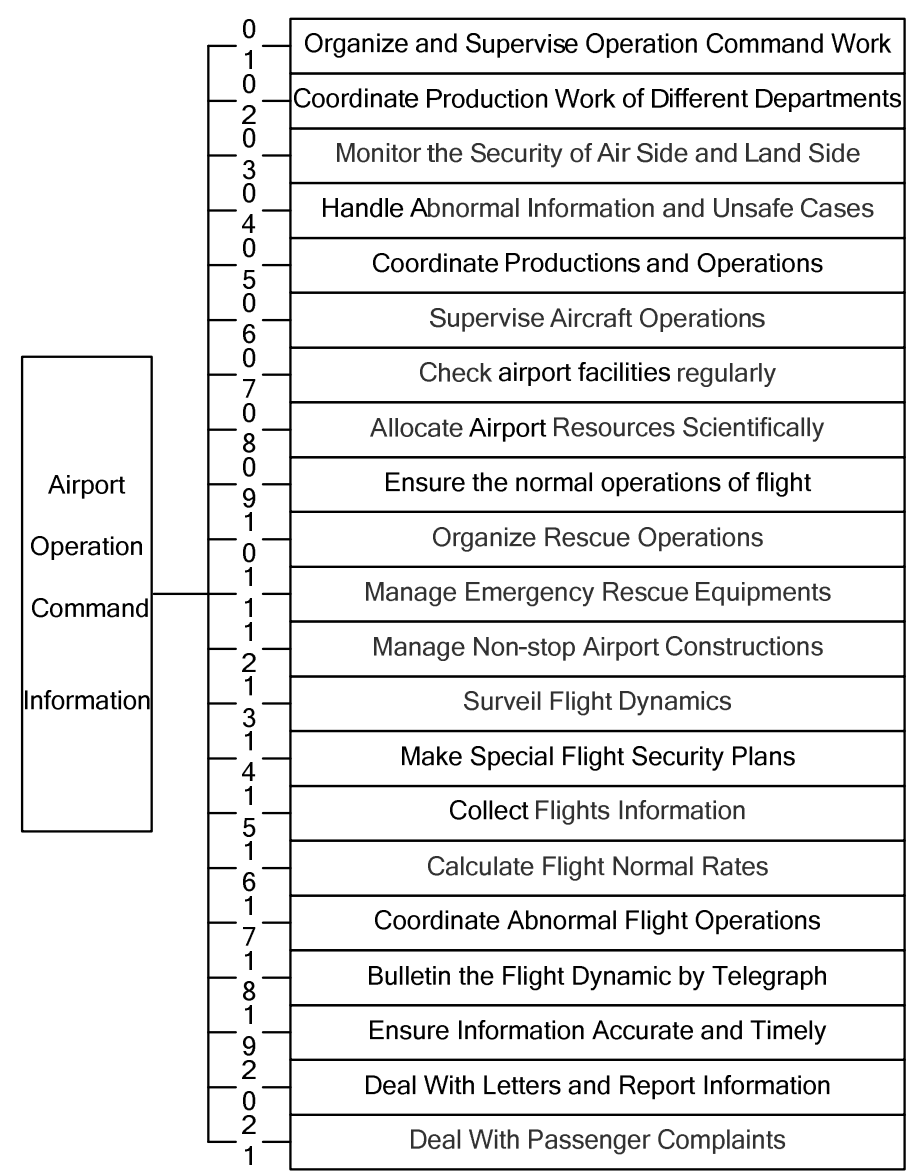

Fig.2 Airport operation command information figure

Establish observation matrix $\mathbf{X}$. Samples, the airport operation command information, having $M$ indexes and $\mathrm{N}$ samples $(\mathrm{M}=7, \mathrm{~N}=21)$, are used to establish the observation matrix:

$$
X=\left[X_{1}, X_{2}, \cdots, X_{M}\right]=\left[\begin{array}{cccc}
x_{11} & x_{12} & \cdots & x_{1 M} \\
x_{21} & x_{22} & \cdots & x_{2 M} \\
\cdots & \cdots & \cdots & \cdots \\
x_{N 1} & x_{N 2} & \cdots & x_{N M}
\end{array}\right]_{N \times M}=\left[\begin{array}{c}
Y_{1} \\
Y_{2} \\
\cdots \\
Y_{N}
\end{array}\right]
$$


Where: the column vector $X_{i}=\left[\begin{array}{c}x_{1 i} \\ x_{2 i} \\ \cdots \\ x_{N i}\end{array}\right](i=1,2, \cdots, M)$ represents the value of the number $\mathrm{i}$ attribute index. The row vector $Y_{r}=\left(x_{r 1}, x_{r 2}, \cdots, x_{r M}\right)(r=1,2, \cdots, N)$ represents the value of the number $r$ sample under the $M$ attribute indexes.

It uses Delphi method to assign the values of sample properties, and establishes the observation matrix X (See Table 1):

Table.1 Observation matrix $\mathrm{X}$ index numerical table

\begin{tabular}{cccccccc}
\hline $\begin{array}{c}\text { Information } \\
\text { Number }\end{array}$ & $\begin{array}{c}\text { Flight } \\
\text { Production }\end{array}$ & $\begin{array}{c}\text { passenger } \\
\text { service }\end{array}$ & $\begin{array}{c}\text { safety } \\
\text { monitoring }\end{array}$ & $\begin{array}{c}\text { facilities } \\
\text { instrument }\end{array}$ & $\begin{array}{c}\text { production } \\
\text { control }\end{array}$ & $\begin{array}{c}\text { emergency } \\
\text { rescue }\end{array}$ & $\begin{array}{c}\text { aeronautical } \\
\text { information }\end{array}$ \\
\hline 01 & 0.25 & 0.1 & 0.3 & 0.2 & 0.25 & 0.3 & 0.2 \\
02 & 0.15 & 0.45 & 0.1 & 0.2 & 0.35 & 0.25 & 0.05 \\
03 & 0.1 & 0.2 & 0.45 & 0.35 & 0.05 & 0.1 & 0.05 \\
04 & 0.2 & 0.15 & 0.35 & 0.25 & 0.2 & 0.35 & 0.2 \\
05 & 0.35 & 0.05 & 0.1 & 0.2 & 0.4 & 0.3 & 0.05 \\
06 & 0.1 & 0.45 & 0.1 & 0.1 & 0.3 & 0.2 & 0.1 \\
07 & 0.05 & 0.15 & 0.4 & 0.45 & 0.05 & 0.1 & 0.05 \\
08 & 0.35 & 0.1 & 0.05 & 0.2 & 0.45 & 0.3 & 0.05 \\
09 & 0.15 & 0.2 & 0.45 & 0.35 & 0.1 & 0.15 & 0.05 \\
10 & 0.15 & 0.1 & 0.3 & 0.25 & 0.2 & 0.35 & 0.15 \\
11 & 0.3 & 0.05 & 0.1 & 0.25 & 0.4 & 0.35 & 0.05 \\
12 & 0.1 & 0.15 & 0.4 & 0.4 & 0.1 & 0.05 & 0.1 \\
13 & 0.3 & 0.2 & 0.1 & 0.05 & 0.1 & 0.05 & 0.65 \\
14 & 0.3 & 0.2 & 0.15 & 0.1 & 0.1 & 0.1 & 0.6 \\
15 & 0.3 & 0.05 & 0.05 & 0.15 & 0.35 & 0.25 & 0.15 \\
16 & 0.25 & 0.15 & 0.1 & 0.05 & 0.05 & 0.05 & 0.55 \\
17 & 0.2 & 0.1 & 0.25 & 0.15 & 0.2 & 0.3 & 0.2 \\
18 & 0.35 & 0.15 & 0.1 & 0.05 & 0.1 & 0.05 & 0.65 \\
19 & 0.1 & 0.4 & 0.15 & 0.15 & 0.3 & 0.2 & 0.05 \\
20 & 0.15 & 0.05 & 0.3 & 0.15 & 0.15 & 0.25 & 0.25 \\
21 & 0.05 & 0.5 & 0.1 & 0.1 & 0.3 & 0.15 & 0.05 \\
\hline
\end{tabular}

Command information clustering. The direct purpose of the airport operation command information clustering is to pick out the information which is in line with the fuzzy evaluation criteria and has high fuzzy similarity from high-dimensional sample space. In this space, each sample object can be regarded as a point in space. It uses distance to characterize their fuzzy similarities. Supposing there are two sample points: $Y_{r}=\left(x_{r 1}, x_{r 2}, \cdots, x_{r M}\right)$ and $Y_{s}=\left(x_{s 1}, x_{s 2}, \cdots x_{s M}\right), r, s=1,2, \cdots, N$,the Euclidean distance between the two points is:

$$
d_{r s}(2)=\left[\sum_{j=1}^{M}\left(x_{r j}-x_{s j}\right)^{2}\right]^{1 / 2}
$$

Next, it uses the average distance method to calculate the distance between classes:

$$
d(r, s)=\frac{1}{n_{r} n_{s}} \sum_{i=1}^{n_{r}} \sum_{j=1}^{n_{s}} \operatorname{dist}\left(x_{r i}, x_{s j}\right)
$$

Where: $n_{r}=n_{s}=N \times(N-1) / 2$, is the distance number between any two observation sample points.

And then we use Matlab, a kind of mathematic software, to cluster the information and to draw cluster pedigree chart (See Figure 3). 


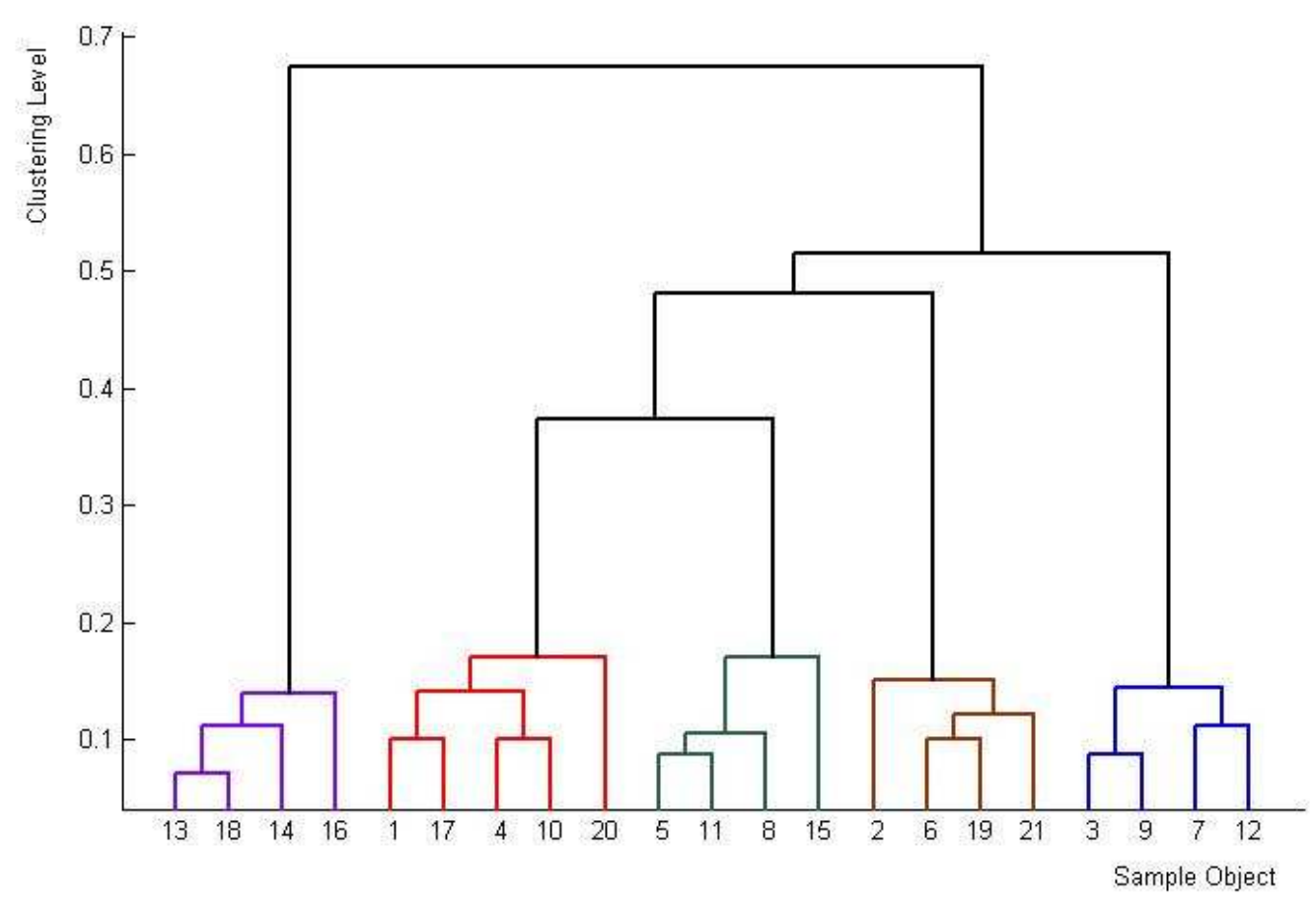

Fig.3 Fuzzy cluster pedigree chart

Clustering effect evaluation. Fuzzy clustering analysis requires pre-determined cluster samples and cluster indexes, and the establishment of the observation matrix, and the final classification which closely related to value of the above data. So it is necessary to evaluate the final outcome of clustering ${ }^{[6]}$. In this paper, it uses the correlation coefficient to judge the clustering results.

$$
c=\frac{\sum_{i<j}\left(Y_{i j}-y\right)\left(Z_{i j}-z\right)}{\sqrt{\sum_{i<j}\left(Y_{i j}-y\right)^{2} \sum_{i<j}\left(Z_{i j}-z\right)^{2}}}
$$

Where: $\mathrm{c}$ is the correlation coefficient, whose value is the closer to 1 , the smaller of ambiguity of the final classification, and the better of the clustering results. $\mathrm{Y}$ is the Euclidean distance between sample points, $Z$ is $(:, 3)$ matrix, whose first two rows are classes, the last one is the distance between classes. $y$ and $z$, are the average value of $Y$ and $Z(:, 3)$ respectively.

It uses Matlab to compute, $\mathrm{c}=0.9189$, which shows the result of the classification is ideal.

\section{The realization of seats distribution}

The general status of the sample classification can be seen through the clustering pedigree chart visually. But with the clustering level, $\lambda$ 's values, changing between $[0,1]$, the clustering results also change accordingly. According to The International Civil Aviation Covenant and accessories 14,-“Airport", technical standards and document requirements about airport operation standardization in the word, experts with rich experience determine $\lambda$ 's value between $[0.2,0.3]$. Having equivalence partition on the level of $\lambda$, we obtain for the clustering results: kind I, $\{1,4,10,17,20\}$; kind II , $\{5,8,11,15\}$; kind III , $\{3,7,9,12\}$; kind IV , $\{2,6,19,21\}$; kind V , $\{13,14,16,18\}$. So it is reasonable for us to divide airport operation command simulation system into 5 positions.

\section{Conclusion}

In this paper, the observation matrix is built based upon the airport operation command information, with the clustering quota of 7 kinds of information attribution. Applying the fuzzy clustering, it distributes the positions of the airport operation command simulation system effectively and clearly. 
This method is practical and reveals different attributes of each seat. Moreover, it overcomes the shortcoming of the traditional distribution methods, and provides a theoretical basis for the positions distribution of the airport operation command simulation system.

1 Received:2012-

Foundation Item: Civil Aviation Authority Technology Projects, The Civil Airport Operation Management Standard Research Plan(RKXZY0723); Research Fund Project of Civil Aviation Flight University of China(X2011-20)

About The Author: Mou Qifeng (1972-), professor in Air Traffic Management Department of China Civil Aviation Flight University, and Ph.D. candidate of Southwest Jiaotong University. His main academic interest is in the field of transportation planning and management, and airport operation management.

Mailing Address: Air Traffic Management Department, Civil Aviation Flight University of China, Guanghan City, Sichuan Prov., China, 618307

\section{References}

[1] Shanghai Hongqiao International Airport. 2008. The Workbook of Moving Command Center Control Room [Z].

[2] Assaf. 2009. Accounting for size in efficiency comparisons of airports [J]. Journal of Air Transport Management, 15, 56-258.

[3] Hans-Martin Niemeier. 2011. The importance of spatial economics for assessing airport competition [J]. Journal of Air Transport Management, 17, 44-48.

[4] Feng Deyi, Lou Shibo. 1983. Fuzzy Mathematics Method and Application [M]. Seismological Press, Beijing (in Chinese).

[5] Xiong Ying. 2008. The Build of airport information integrated system under mult-terminal operating mode [J]. Science \& Technology Information, 33, 3-4.

[6] Yin Yong, Zhan Shuguang, Liu Wenli. 2011. Study on Chinese new marshalling station classification [J]. Journal of Transportation Engineering and Information, 3, 22-25. 